\title{
On lower eigenvalue bounds for Toeplitz operators with radial symbols in Bergman spaces
}

\author{
Grigori Rozenblum
}

\begin{abstract}
We consider Toeplitz operators in different Bergman type spaces, having radial symbols with variable sign. We show that if the symbol has compact support or decays rapidly, the eigenvalues of such operators cannot decay too fast, essentially faster than for a sign-definite symbol of the same kind. On the other hand, if the symbol decays not sufficiently rapidly, the eigenvalues of the corresponding operator may decay faster than for the operator corresponding to the absolute value of the symbol.
\end{abstract}

Mathematics Subject Classification (2010). Primary 47B35; Secondary: 47A75, 46E22, $30 \mathrm{H} 20$.

Keywords. Bergman spaces, Bargmann spaces, Toeplitz operators.

\section{Introduction}

Toeplitz operators arise in many fields of Analysis. The general setting is the following. Let $\mathscr{H}$ be a Hilbert space of functions and $\mathscr{B}$ be a closed subspace in $\mathscr{H}$. For a function $V$, called the symbol further on, the Toeplitz operator $T_{V}: \mathscr{B} \rightarrow \mathscr{B}$ acts as $T_{V}: u \mapsto P V u$, where $P$ is the orthogonal projection from $\mathscr{H}$ onto $\mathcal{B}$. Of course, it is supposed that the operator of multiplication by $V$ maps $\mathcal{B}$ into $\mathcal{H}$.

In the present paper we consider Toeplitz operators in some Bergman type spaces. Let $\Omega$ be a domain in $\mathbb{R}^{d}$ or $\mathbb{C}^{d}, \mathscr{H}$ be the space $L^{2}(\Omega)$ with respect to some measure $\mu$, and $\mathscr{B}$ be the subspace in $\mathscr{H}$ consisting of solutions of some elliptic equation or system. The leading example here is provided by Bergman-Toeplitz operators, where $\Omega$ is a bounded domain with nice boundary and $\mathcal{B}$ consists of harmonic functions in $\mathscr{H}$ (the harmonic Bergman space), and, in the complex case, $\mathscr{B}$ consists of analytical functions in $\Omega$ (the analytical Bergman space). Another series of examples is given by Bargmann-Toeplitz, operators where $\Omega$ is the whole (real or complex) space, $\mathscr{H}=L^{2}(\Omega)$ with respect to the Gaussian measure, and $\mathscr{B}$ consists of harmonic or (in the complex case) analytical functions in $\mathcal{H}$.

We are interested in the spectral properties of Toeplitz operators for the case when the symbol $V$, which is supposed to be real and bounded, has compact support (when 
$\Omega$ is a ball) or decays rapidly at infinity (when $\Omega$ is the whole space). One can easily see that such operator is compact, and our question is about determining how fast the eigenvalues of $T_{V}$ tend to zero. The interest to this topic grew recently due to the close relation of the spectral properties of Toeplitz operators to the spectral analysis of the perturbed Landau Hamiltonian describing the quantum particle in a homogeneous magnetic field.

For a sign-definite symbol, in the complex Bargmann case, rather complete results were obtained in [15] and [12] and, in dimension $d=1$, improved in [6], see also references therein. Even earlier, the case of the complex Bergman space in dimension $d=1$ has been studied in [13]. It was proved that the eigenvalues of the Toeplitz operator follow an asymptotic law, of an exponential type for the Bergman case and super-exponential type for the Bargmann one.

For the case of the symbol $V$ having variable sign, it was for a long time unclear, even whether it is possible that the positive and negative parts $V_{ \pm}$of $V$ can compensate each other almost completely, so that the spectrum of $T_{V}$ is finite. It has been proved only recently, see [8], that such complete cancelation is impossible, in other words, for a nontrivial symbol $V$ with compact support the Toeplitz operator cannot have finite rank; see [16] for the most complete results on the finite rank problem and related references. A further analysis in [14] has shown that the (infinite now) spectrum of the Toeplitz operator depends essentially on the geometry of the support of $V_{ \pm}$. In particular, if, say, the support of $V_{+}$surrounds the support of $V_{-}$(in a proper meaning), then the negative spectrum of $T_{V}$ is finite and the asymptotic behavior of the positive eigenvalues is the same as if $V_{-}$was absent. On the other hand, if $V_{ \pm}$are supported in geometrically well separated sets, then both the positive and the negative spectra of $T_{V}$ are infinite and, put together, obey the same asymptotic law as if $V$ was sign-definite (in the Bargmann case); these results can be understood as showing that no cancelation of $V_{ \pm}$takes place for this class of symbols, as it concerns the asymptotic properties of the spectrum.

In the present paper we continue the study of the spectrum of Toeplitz operators with non-sign-definite symbol. We consider the model case of the symbol $V$ being radial, i.e. depending only on the the distance to the origin; no restrictions on the supports of $V_{ \pm}$are imposed. We find out that there is an essential difference in spectral properties of Toeplitz operators with rapidly decaying symbols (including compactly supported ones, the exact definitions are given in the paper), on the one hand, and symbols decaying not that rapidly, on the other. In the former case we establish that, although no information on the positive and negative spectra of $T_{V}$ can be obtained separately, the distribution function of the positive and negative eigenvalues counted together, i.e. of singular numbers of the operator, is subject to lower asymptotical bounds that have the same order and even the same coefficient as if the symbol was sign definite. This is expressed, in a general form, by the relation (4.1). So, again, no cancelation happens. On the other hand, in the latter case it is possible that the eigenvalues of the Toeplitz operator decay considerably faster than for the operator with the corresponding sign-definite symbol. 
We start in Section 2 with describing the Bergman type spaces under consideration (we consider operators in the Bergman and Bargmann spaces of analytical and harmonic functions as well as in the spaces of solutions of the Helmholtz equation) and finding the expression for the eigenvalues. These expressions are quite explicit. The asymptotic formulas for eigenvalues are obtained, first for $V$ being the characteristic function of a ball, and then these results are carried over to general sign-definite compactly supported case by means of simple monotonicity arguments. For Bargmann spaces a class of symbols with rapid decay at infinity is considered as well. Some of these results are well known, the remaining are obtained in a more or less standard way - we, however, present them all here for further reference.

Passing to general non-sign-definite radial symbols, we encounter a serious inconvenience. We still can write the explicit expression for the eigenvalues, however the numbering of the eigenvalues in the non-increasing or in the non-decreasing order does not coincide with their natural numbering, stemming from the one in the separation of variables, and the relation between two numberings is rather hard to control. To handle this circumstance, we need certain considerations from infinite combinatorics (Proposition 3.1). In order to prove that the eigenvalues cannot decay too fast, we need rather advanced results in complex analysis, and these results are also presented in Section 3. The main results of the paper on the lower eigenvalue estimates for general non-sign-definite radial symbols are presented in Section 4, see Theorems 4.1, 4.2. Finally, in Section 5, we describe examples showing that a considerable cancelation may take place for not sufficiently rapidly decaying fast oscillating symbols.

\section{Eigenvalues of Toeplitz operators with radial symbols}

In this Section we calculate the eigenvalue asymptotics for Toeplitz operators with radial symbols in the spaces under consideration. For sign-definite symbols some of these results are known, others are obtained in a standard way using the explicit expressions for the eigenvalues.

2.1. Eigenvalues and reordering -1 . For each of operators $T_{V}$ under consideration in the paper, we are going to find explicitly the sequence of eigenvalues $\Lambda_{k}$ having multiplicities $\mathbf{d}_{k}$. To describe the ordered set of eigenvalues, counting multiplicities, one should consider the set of the numbers $\Lambda_{k}$, each $\Lambda_{k}$ counted $\mathbf{d}_{k}$ times, and then reorder the positive numbers in the non-increasing way and the negative ones in the non-decreasing way. Thus we obtain two (finite or infinite) sequences $\lambda_{n}^{ \pm}$of eigenvalues of $T_{V}$. The union of the sequences $\pm \lambda_{n}^{ \pm}$is the sequence of $s$-numbers, numerated in the nonincreasing order: $s_{n}=s_{n}\left(T_{V}\right)$.

For the problems under consideration, it is often more convenient to describe the 
spectrum by means of the counting functions defined as

$$
n_{ \pm}(\lambda)=\#\left\{n: \pm \lambda_{n}^{ \pm}>\lambda\right\}, \quad n(\lambda)=n_{+}(\lambda)+n_{-}(\lambda)=\#\left\{n: s_{n}>\lambda\right\}
$$

we will include the designation of the operator and the space in question in the notation, when needed. In the terms of multiplicities, we, obviously, have

$$
n_{ \pm}(\lambda)=\sum_{ \pm \Lambda_{k}>\lambda} \mathbf{d}_{k}, \quad n(\lambda)=\sum_{\left|\Lambda_{k}\right|>\lambda} \mathbf{d}_{k} .
$$

In the most simple cases below, the sequence $\Lambda_{k}$ is non-negative and already non-increasing, and thus no reordering is needed. However, generally, we should not expect that the sequence $\left|\Lambda_{k}\right|$ is monotonous, and thus the question arises, how the estimates for $\left|\Lambda_{k}\right|$ are related to estimates of this sequence monotonously reordered. In one direction, the result is obvious. We, however, formulate it in order to be able to refer to it later on.

Proposition 2.1. Let $a_{k}, b_{k}, k=0,1, \ldots$, be two sequence of real numbers, so that $b_{k}>0, b_{k} \rightarrow 0$ monotonously. Suppose that $\left|a_{k}\right| \leq b_{k}$. Denote by $a_{k}^{*}$ the sequence obtained by the non-increasing reordering of the sequence $\left|a_{k}\right|$. Then $a_{k}^{*} \leq b_{k}$.

Of course, generally, one should not expect the direct conversion of Proposition 2.1 to be correct: an estimate for the monotonously reordered sequence cannot be carried over to the initial sequence. It turns out, however, that in a certain sense Proposition 2.1 can be partially conversed, see Proposition 3.3.

The operators we consider in this paper have very fast, exponential or even superexponential rate of decay of eigenvalues. Since these eigenvalues have very high multiplicities, the eigenvalues $\lambda_{n}^{ \pm}$, counting their multiplicities, do not follow any regular asymptotic law, unlike the well-studied case of elliptic operators. Therefore it is more convenient to consider the behavior of the eigenvalues in the logarithmic scale, where the oscillations caused by high multiplicities are suppressed and a regular asymptotics exists. In this scale, in particular, the leading term of the asymptotics does not change when the symbol $V$ (and thus the eigenvalues of the Toeplitz operator) is multiplied by a positive constant. Alternatively, the asymptotical behavior of the eigenvalues can be described by their counting function. Unlike the case of a powerlike asymptotics, typical for elliptic boundary problems, the asymptotic formula for the counting function is not equivalent to the one for the eigenvalues, however it is equivalent to the asymptotic eigenvalue formula in the logarithmic scale. We will use this equivalence persistently.

We will use the following notation. For functions $f$ and $g$ of a real or integer argument $t$, the symbol $f(t) \sim g(t)$ means, as usual, $f(t) / g(t) \rightarrow 1$ as $t \rightarrow \infty$ or $t \rightarrow 0$, which is always clear from the context. The relation $f(t) \lesssim g(t)$ means that $\lim \sup f(t) / g(t) \leq 1$, the obvious meaning has the notation $f(t) \gtrsim g(t)$. Finally, $f(t) \asymp g(t)$ is used when $c f(t) \leq g(t) \leq C f(t)$ for sufficiently large (or small) $t$ and for some positive constants $c, C$. 


\subsection{Bergman type spaces and quadratic forms}

2.2.1. The spaces. The following Bergman type spaces will be considered in this paper.

(1) Bergman spaces.

- The Bergman spaces of analytical functions in the ball. Let $D^{2 d} \subset \mathbb{C}^{d}, d \geq 1$ be the ball $|z|<\mathbf{R}$ with radius $\mathbf{R}$. The space $\mathscr{H}$ is the space $L^{2}\left(D^{2 d}\right)$ with respect to the Lebesgue measure and $\mathscr{B}=\mathscr{B}_{\mathbf{R}}^{\mathbb{C}} \subset \mathcal{H}$ consists of analytical functions.

Remark 2.2. In the literature, Bergman spaces with the Lebesgue measure with weight $\left(1-(|z| / \mathbf{R})^{2}\right)^{\alpha}$ are considered as well. The results of the paper extend to this case almost automatically.

- The Bergman spaces of harmonic functions in the ball. Let $D^{d} \subset \mathbb{R}^{d}, d>1$, be the ball with radius $\mathbf{R}$. The space $\mathscr{H}$ is $L^{2}\left(D^{d}\right)$ with respect to the Lebesgue measure and $\mathscr{B}=\mathscr{B}_{\mathbf{R}}^{\mathbb{R}} \subset \mathscr{H}$ consists of harmonic functions.

- The Bergman spaces of solutions of the Helmholtz equation. The space $\mathscr{H}$ is, again, $L^{2}\left(D^{d}\right)$ and $\mathscr{B}=\mathscr{B}_{\mathbf{R}}^{\mathbf{H}} \subset \mathcal{H}$ consists of solutions of the Helmholtz equation $\Delta u+u=0$.

(2) Bargmann spaces.

- The Bargmann spaces of analytical functions in $\mathbb{C}^{d}$. Here $\mathscr{H}$ is $L^{2}\left(\mathbb{C}^{d}\right)$ with respect to the Lebesgue measure with Gaussian weight $e^{-|z|^{2}}$ and $\mathscr{B}=\mathscr{B}^{\mathbb{C}} \subset \mathscr{H}$ consists of analytical functions.

- The Bargmann spaces of harmonic functions in $\mathbb{R}^{d}$. Here $\mathscr{H}$ is $L^{2}\left(\mathbb{R}^{d}\right)$ with respect to the Lebesgue measure with Gaussian weight $e^{-|x|^{2}}$ and $\mathscr{B}=\mathscr{B}^{\mathbb{R}} \subset \mathscr{H}$ consists of harmonic functions.

- The Bargmann spaces of solutions of the Helmholtz equation in $\mathbb{R}^{d}$. Here $\mathscr{H}$ is $L^{2}\left(\mathbb{R}^{d}\right)$ with respect to the Lebesgue measure with Gaussian weight $e^{-|x|^{2}}$ and $\mathscr{B}=\mathscr{B}^{\mathrm{H}} \subset \mathcal{H}$ consists of solutions of the Helmholtz equation.

(3) The Agmon-Hörmander space $\mathscr{H}=B^{*}$, see [1] and [19], is defined as consisting of (equivalence classes of) functions $u \in L_{\text {loc }}^{2}\left(\mathbb{R}^{d}\right)$ such that the norm

$$
\|u\|_{B^{*}}=\left(\sup _{r \in(0, \infty)} r^{-1} \int_{|x|<r}|u|^{2} d x\right)^{\frac{1}{2}}
$$

is finite. All functions $u$ in this space, satisfying the Helmholtz equation, form a closed subspace $\mathscr{B}=\mathscr{B}^{\mathbf{A H}}$. For $u \in \mathscr{B}^{\mathbf{A H}}$, the limit

$$
\||| u||^{2}=\lim _{r \rightarrow \infty} r^{-1} \int_{|x|<r}|u|^{2} d x
$$

exists, and it defines a norm equivalent to (2.2), see [19], Lemma 3.2. 
2.2.2. Toeplitz operators and quadratic forms. The convenient way to study the eigenvalues of Toeplitz operators is by using the quadratic form setting. Let $\mathscr{B} \subset \mathcal{H}$ be the Bergman type space under study and $\langle\cdot, \cdot\rangle$ and $\|\cdot\|$ be the corresponding scalar product and norm. The Toeplitz operator $T_{V}$ in $\mathscr{B}$ is defined by the quadratic form $h_{V}[u]=\langle V u, u\rangle, u \in \mathscr{B}$. It is convenient to use this definition even in the case when one does not consider the embracing space, as, for example, for $\mathscr{B}=\mathscr{B}^{\mathbf{A H}}$ : having a Bergman type space $\mathscr{B}$ we will still call the operator $T_{V}$ defined by the quadratic form $\int V|u|^{2} d \mu$ in $\mathscr{B}$ the Toeplitz operator in $\mathscr{B}$ with symbol $V$. As soon as a complete system of functions $u_{n} \in \mathscr{B}$ is found, which diagonalizes both quadratic forms $\|u\|^{2}$ and $h_{V}[u]$, this system can serve as a complete system of eigenfunctions of $T_{V}$, with eigenvalues $h_{V}\left[u_{n}\right] /\left\|u_{n}\right\|^{2}$. We emphasize here again that these eigenvalues should be properly reordered.

In this paper we are going to study Toeplitz operators with radial symbols, i.e. $V(z)=V(|z|)$ in the complex case and $V(x)=V(|x|)$ in the real case. For such symbols the eigenfunctions and eigenvalues of the Toeplitz operator can be found explicitly by means of passing to spherical coordinates.

2.3. Operators in Bergman spaces. In the sections to follow we collect the results on the eigenvalue asymptotic formulas for Toeplitz operators in Bergman spaces. Some of them are already known, the rest are obtained in a standard way, using the explicit expressions for the eigenvalues.

2.3.1. Complex Bergman spaces. Denote by $\mathcal{P}_{k}^{\mathbb{C}}$ the space of homogeneous polynomials of degree $k$ of the variables $z_{1}, \ldots, z_{d}$. Its dimension is $\mathbf{d}_{k}^{\mathbb{C}}=\left(\begin{array}{c}k+d-1 \\ d-1\end{array}\right)=$ $\frac{k^{d-1}}{(d-1) !}\left(1+O\left(k^{-1}\right)\right)$. In the space of functions of the form $Z(\omega)=p(z)|z|^{-k}$, $p \in \mathcal{P}_{k}^{\mathbb{C}}, \omega=z|z|^{-1} \in S^{2 d-1}$, we choose a basis $Z_{k, j}(\omega), j \in\left[1, \mathbf{d}_{k}^{\mathbb{C}}\right]$, orthonormal with respect to the Lebesgue measure on the sphere $S^{2 d-1}$ (the complex spherical functions). The functions $u_{k, j}(z)=|z|^{k} Z_{k, j}(\omega), k=0,1, \ldots, j \in\left[1, \mathbf{d}_{k}^{\mathbb{C}}\right]$, form an orthogonal basis in the space $\mathcal{B}_{\mathbf{R}}^{\mathbb{C}}$. For a radial function $V(|z|)$, this system of functions diagonalizes also the quadratic form $\int V(|z|)|u(z)|^{2} d \mu$. Therefore, the functions $u_{k, j}$ form a complete system of eigenfunctions of the Toeplitz operator $T_{V}$ in $\mathscr{B}_{\mathbf{R}}^{\mathbb{C}}$ with the eigenvalues

$$
\begin{aligned}
\Lambda_{k}=\Lambda_{k}^{\mathbb{C}}(V) & =\frac{\left\langle V u_{k, j}, u_{k, j}\right\rangle}{\left\|u_{k, j}\right\|_{\mathcal{B}_{\mathbf{R}}^{\mathbb{C}}}^{2}} \\
& =(2 k+2 d) \mathbf{R}^{-(2 k+2 d)} \int_{0}^{\mathbf{R}} V(r) r^{2 k+2 d-1} d r,
\end{aligned}
$$

having multiplicity $\mathbf{d}_{k}^{\mathbb{C}}$. By reordering, see Section 2.1, we obtain two sequences $\lambda_{n}^{ \pm}$of eigenvalues of $T_{V}$. By the results of [2], see also [16], at least one of these sequences is infinite. 
Let $V$ be the characteristic function of the ball $D_{b}$ with center at the origin and radius $b \in(0, \mathbf{R})$. Then there are no negative eigenvalues, and the positive eigenvalues, by (2.3), are $\Lambda_{k}=(b / \mathbf{R})^{2 k+2 d}$ with multiplicities $\mathbf{d}_{k}^{\mathbb{C}}$. Taking into account the asymptotics $\mathbf{d}_{k}^{\mathbb{C}} \sim k^{d-1} /(d-1)$ !, we have in terms of the counting function,

$$
\begin{aligned}
n\left(\lambda ; T_{V}, \mathscr{B}_{\mathbf{R}}^{\mathbb{C}}\right) & \sim \sum_{(b / \mathbf{R})^{2 k+2 d}>\lambda} \frac{k^{d-1}}{(d-1) !} \\
& \sim(d !)^{-1}(2|\log (b / \mathbf{R})|)^{-d}|\log \lambda|^{d}, \quad \lambda \rightarrow 0,
\end{aligned}
$$

or, in the logarithmic scale,

$$
\log \left(\lambda_{n}^{+}\right)=\log \left(s_{n}\left(T_{V}\right)\right) \sim 2(n d !)^{\frac{1}{d}} \log (b / \mathbf{R}) .
$$

2.3.2. Harmonic Bergman spaces. Denote by $\mathcal{P}_{k}^{\mathbb{R}}$ the space of degree $k$ homogeneous harmonic polynomials of the variables $x_{1}, \ldots, x_{d}$. This space has dimension $\mathbf{d}_{k}^{\mathbb{R}}=\left(\begin{array}{c}d+k-1 \\ d-1\end{array}\right)-\left(\begin{array}{c}d+k-2 \\ d-2\end{array}\right)=\frac{2}{(d-2) !} k^{d-2}\left(1+O\left(k^{-1}\right)\right)$, see, e.g., the calculations in [17], Section 22. In the space of functions of the form $Y(\omega)=p(x)|x|^{-k}, p \in \mathcal{P}_{k}^{\mathbb{R}}$, $\omega=x|x|^{-1} \in S^{d}$, we choose a basis $Y_{k, j}(\omega), j \in\left[1, \mathbf{d}_{k}^{\mathbb{R}}\right]$, orthonormal with respect to the Lebesgue measure on the sphere $S^{d-1}$, i.e. the usual spherical functions. The functions $u_{k, j}(x)=|x|^{k} Y_{k, j}(\omega), k=0,1, \ldots, j \in\left[1, \mathbf{d}_{k}^{\mathbb{R}}\right]$, form an orthogonal basis in the space $\mathcal{B}_{\mathbf{R}}^{\mathbb{R}}$. For a radial function $V(|x|)$, this system of functions diagonalizes also the quadratic form $\int V(|x|)|u(x)|^{2} d \mu$. Therefore, the functions $u_{k, j}$ form a complete system of eigenfunctions of the Toeplitz operator $T_{V}$ in $\mathcal{B}_{\mathbf{R}}^{\mathbb{R}}$ with the eigenvalues $\Lambda_{k}^{\mathbb{R}}$ given by

$$
\Lambda_{k}=\Lambda_{k}^{\mathbb{R}}(V)=(2 k+d) \mathbf{R}^{-2 k-d} \int_{0}^{\mathbf{R}} V(r) r^{2 k+d-1} d r
$$

and multiplicities $\mathbf{d}_{k}^{\mathbb{R}}$. Taking into account the multiplicities and reordering, as in Section 2.3.1, we obtain the eigenvalue sequences $\lambda_{n}^{ \pm}$and the sequence of $s$-numbers $s_{n}$. Again, as it is shown in [2], the sequence $s_{n}$ and at least one of the sequences $\lambda_{n}^{ \pm}$ are infinite.

For $V$ being the characteristic function of the ball $D_{b}$, the eigenvalues $\Lambda_{k}^{\mathbb{R}}$ are equal to $\Lambda_{k}^{\mathbb{R}}=(b / \mathbf{R})^{2 k+d}$, by (2.6). Thus, there are no negative eigenvalues $\lambda_{n}^{-}$, while for the positive eigenvalues $\lambda_{n}^{+}$we have the asymptotics

$$
\begin{aligned}
n\left(\lambda ; T_{V}, B_{\mathbf{R}}^{\mathbb{R}}\right) & \sim \sum_{(b / \mathbf{R})^{2 k+d}>\lambda} 2 \frac{k^{d-2}}{(d-2) !} \\
& \sim 2((d-1) !)^{-1}(2|\log (b / \mathbf{R})|)^{-d+1}|\log \lambda|^{d-1}, \quad \lambda \rightarrow 0,
\end{aligned}
$$

and, in the logarithmic scale,

$$
\log \lambda_{n}^{+} \sim 2 \log (b / \mathbf{R})((d-1) ! / 2)^{\frac{1}{d-1}} n^{\frac{1}{d-1}}, \quad n \rightarrow \infty .
$$


2.3.3. Helmholtz Bergman spaces. After passing to spherical coordinates in the Helmholtz equation, we arrive at the orthogonal system of functions

$$
u_{k, j}(x)=Y_{k, j}(\omega)|x|^{-\frac{d-2}{2}} J_{k+\frac{d-2}{2}}(|x|), \quad \omega=x|x|^{-1} \in S^{d-1},
$$

where $k=0,1, \ldots, j=1, \ldots, \mathbf{d}_{k}^{\mathbb{R}}, J_{v}(r)$ are the Bessel functions and $Y_{k, j}$ are the real spherical functions as in Section 2.3.2. For a radial symbol $V(|x|)$, the eigenvalues of the Toeplitz operator equal

$$
\Lambda_{k}=\Lambda_{k}^{\mathbf{H}}\left(T_{V}\right)=\frac{\int_{0}^{\mathbf{R}} V(r) J_{k+\frac{d-2}{2}}^{2}(r) r d r}{\int_{0}^{\mathbf{R}} J_{k+\frac{d-2}{2}}^{2}(r) r d r},
$$

with the multiplicity $\mathbf{d}_{k}^{\mathbb{R}}$. The integral in the denominator in (2.9) is estimated by means of the identity, see, e.g., [20],

$$
\int_{0}^{R} J_{v}^{2}(r) r d r=\frac{R^{2}}{2}\left[J_{v}^{2}(R)-J_{v-1}(R) J_{v+1}(R)\right],
$$

and the asymptotics, see again [20], uniform in $r$ on any finite interval $[a, b] \subset[0, \infty)$ :

$$
J_{v}(r) \sim\left(\frac{r^{2}}{2}\right)^{v}(\Gamma(v+1))^{-1}, \quad|v| \rightarrow+\infty, \quad \operatorname{Re} v \geq 0 .
$$

So, we obtain

$$
\int_{0}^{\mathbf{R}} J_{k+\frac{d-2}{2}}^{2}(r) r d r \sim\left(\frac{\mathbf{R}^{2}}{2}\right)^{\frac{k+d}{2}} \frac{1}{\Gamma\left(k+\frac{d}{2}\right) \Gamma\left(k+\frac{d+2}{2}\right)}, \quad k \rightarrow \infty .
$$

Again, as before, the numbers $\Lambda_{k}^{\mathbf{H}}$, counted with multiplicities $\mathbf{d}_{k}^{\mathbb{R}}$ and properly reordered, form the sequences $\lambda_{n}^{ \pm}=\lambda_{n}^{ \pm}\left(T_{V}\right)$ of eigenvalues of $T_{V}$, and the union of the sequences $\pm \lambda_{n}^{ \pm}$is the sequence of $s$-numbers $s_{n}=s_{n}\left(T_{V}\right)$. It is proved in [16] that for $d>2$ at least one of the sequences $\lambda_{n}^{ \pm}$is infinite. The proof in [16] does not cover the case $d=2$, and the above infiniteness will follow from the results of the present paper.

For $V$ being the characteristic function of the ball $|x| \leq b<\mathbf{R}$, the numbers $\Lambda_{k}^{\mathbf{H}}(V)$ have, by (2.10), (2.11), and (2.12), the asymptotics

$$
\Lambda_{k}^{\mathbf{H}} \sim(b / \mathbf{R})^{2 k+d} .
$$

Therefore, taking into account the multiplicities, the eigenvalues $\lambda_{n}^{+}$obey the asymptotic law (2.7)-(2.8), the same as for the harmonic Bergman space. 


\subsection{Operators in Bargmann and AH spaces}

2.4.1. Complex Bargmann spaces. The functions $u_{k, j}(z)=Z_{k, j}(\omega)|z|^{k}, j \in$ $\left[1, \mathbf{d}_{k}^{\mathbb{C}}\right]$, form an orthogonal basis in the Bargmann space $\mathscr{B}^{\mathbb{C}}$. Thus, the eigenvalues of the Toeplitz operator $T_{V}$ in $\mathscr{B}^{\mathbb{C}}$ equal

$$
\Lambda_{k}^{\mathbb{C}}=\frac{\int_{0}^{\infty} V(r) r^{2 k+2 d-1} e^{-r^{2}} d r}{\int_{0}^{\infty} r^{2 k+2 d-1} e^{-r^{2}} d r}=2 \frac{\int_{0}^{\infty} V(r) r^{2 k+2 d-1} e^{-r^{2}} d r}{\Gamma(k+d)} .
$$

For the case of $V(r)$ being the characteristic function of $D_{b}, 0<b<\infty$, obviously,

$$
\left|\log \int_{0}^{\infty} V(r) r^{2 k+2 d-1} e^{-r^{2}} d r\right| \asymp k,
$$

and, therefore, by the Stirling formula,

$$
\left|\log \Lambda_{k}^{\mathbb{C}}\right| \sim k \log k .
$$

Taking into account the multiplicities, we obtain for the eigenvalues of $T_{V}$ :

$$
n(\lambda)=\sum_{\Lambda_{k}^{\mathbb{C}}>\lambda} \mathbf{d}_{k}^{\mathbb{C}} \sim \frac{1}{d !}\left(\frac{|\log \lambda|}{\log |\log \lambda|}\right)^{d},
$$

or, in the logarithmic scale, inverting (2.15):

$$
\log \left(\lambda_{n}^{+}\right)=\log \left(s_{n}\right) \sim-d^{-1}(d !)^{\frac{1}{d}} n^{\frac{1}{d}} \log n .
$$

Remark 2.3. The asymptotic relation (2.15) was found in [12] (in [15] for $d=1$ ); it was discovered there, in particular, that the leading term in the eigenvalue asymptotics of Bargmann-Toeplitz operators does not depend on the symbol $V \geq 0$ with compact support (of course, provided it is not identically zero). In [6], for $d=1$, the second term of the asymptotics in (2.16) was found, depending on the logarithmic capacity of $\operatorname{supp} V$.

2.4.2. Harmonic Bargmann spaces. The functions $u_{k, j}(x)=Y_{k, j}(\omega)|x|^{k}, j \in$ $\left[1, \mathbf{d}_{k}^{\mathbb{R}}\right]$, form an orthogonal basis in the Bargmann space $\mathscr{B}^{\mathbb{R}}$. Thus, the eigenvalues of the Toeplitz operator $T_{V}$ in $\mathscr{B}^{\mathbb{R}}$ equal

$$
\Lambda_{k}^{\mathbb{R}}=\frac{\int_{0}^{\infty} V(r) r^{2 k+d-1} e^{-r^{2}} d r}{\int_{0}^{\infty} r^{2 k+d-1} e^{-r^{2}} d r}=2 \frac{\int_{0}^{\infty} V(r) r^{2 k+d-1} e^{-r^{2}} d r}{\Gamma\left(k+\frac{d}{2}\right)} .
$$


For $V$ being the characteristic function of $D_{b}$ we obtain for the eigenvalues of $T_{V}$, taking into account the multiplicities:

$$
n(\lambda)=\sum_{\Lambda_{k}^{\mathbb{R}}>\lambda} \mathbf{d}_{k}^{\mathbb{R}} \sim 2((d-1) !)^{-1}\left(\frac{|\log \lambda|}{\log |\log \lambda|}\right)^{d-1},
$$

or, in the logarithmic scale, inverting (2.17),

$$
\log \left(\lambda_{n}^{+}\right)=\log \left(s_{n}\right) \sim-(d-1)^{-1}((d-1) ! / 2)^{\frac{1}{d-1}}\left(n^{\frac{1}{d-1}} \log n\right), \quad n \rightarrow \infty .
$$

2.4.3. Helmholtz Bargmann spaces. The functions

$$
u_{k, j}(x)=Y_{k, j}(\omega)|x|^{-\frac{d-2}{2}} J_{k+\frac{d-2}{2}}(|x|) \quad k=0,1, \ldots, \quad j=1, \ldots, \mathbf{d}_{k}^{\mathbb{R}},
$$

form an orthogonal basis in the space $\mathscr{B}^{\mathrm{H}}$. Thus, the eigenvalues of $T_{V}$ in $\mathscr{B}^{\mathrm{H}}$ equal

$$
\Lambda_{k}=\Lambda_{k}^{\mathbf{H}}(V)=\frac{\int_{0}^{\infty} V(r) J_{k+\frac{d-2}{2}}(r)^{2} r e^{-r^{2}} d r}{\int_{0}^{\infty} J_{k+\frac{d-2}{2}}(r)^{2} e^{-r^{2}} r d r},
$$

with multiplicity $\mathbf{d}_{k}^{\mathbb{R}}$. The denominator in (2.19) equals $\frac{1}{2} \exp \left(-\frac{1}{2}\right) I_{k+\frac{d-1}{2}}\left(\frac{1}{2}\right)$, where $I_{\nu}$ is the modified Bessel function (see [7], 6.663.2). By (2.11), this denominator has the asymptotics $\left(\frac{1}{2}\right)^{k+\frac{d-1}{2}} \exp \left(-\frac{1}{2}\right) \Gamma\left(k+\frac{d+1}{2}\right)^{-1}$.

For $V$ being the characteristic function of the ball $D_{b}$, the numerator in (2.19) is estimated from above and from below by constants times $b^{2 k+d}\left(\Gamma\left(k+\frac{d}{2}\right) \Gamma(k+\right.$ $\left.\left.\frac{d+1}{2}\right)\right)^{-1}$. Therefore, in this case, the eigenvalues $\Lambda_{k}^{\mathbf{H}}$ obey two-sided asymptotic estimates

$$
\Lambda_{k}^{\mathbf{H}} \asymp\left(\frac{1}{2}\right)^{k+\frac{d}{2}} b^{2 k+d}\left(\Gamma\left(k+\frac{d+1}{2}\right)\right)^{-1} .
$$

Taking into account the multiplicities, the eigenvalues of the Toeplitz operator $T_{V}$ in the space $\mathscr{B}^{\mathbf{H}}$ have the same asymptotics (2.17)-(2.18) as for the harmonic Bargmann space.

2.4.4. The Agmon-Hörmander space. The functions

$$
u_{k, j}(x)=Y_{k, j}(\omega)|x|^{-\frac{d-2}{2}} J_{k+\frac{d-2}{2}}(|x|), \quad k=0,1, \ldots, \quad j=1, \ldots, \mathbf{d}_{k}^{\mathbb{R}},
$$

form an orthogonal basis in the space $\mathscr{B}^{\mathbf{A H}}$. The $\mathbf{A H}$-norm of these functions equals $\frac{1}{\pi}$ (see, e.g., [19], p. 63). Thus, the eigenvalues of the Toeplitz operator $T_{V}$ in $\mathscr{B}^{\mathrm{AH}}$ equal

$$
\Lambda_{k}^{\mathrm{AH}}=\pi \int_{0}^{\infty} V(r) J_{k+\frac{d-2}{2}}(|r|)^{2} r d r .
$$


For $V$ being the characteristic function of $D_{b}$, these eigenvalues have the asymptotics

$$
\Lambda_{k}^{\mathrm{AH}} \sim \pi\left(\frac{b^{2}}{2}\right)^{k+\frac{d-2}{2}}\left(\Gamma\left(k+\frac{d}{2}\right)\right)^{-2} .
$$

So, the eigenvalues of the Toeplitz operator in the space $\mathscr{B}^{\mathrm{AH}}$ decay considerably faster than in the space $\mathscr{B}^{\mathbf{H}}$, with the same symbol. Counting multiplicities, we obtain for the eigenvalues of $T_{V}$ the asymptotics

$$
\log \lambda_{n}=\log \left(s_{n}\right) \sim-\frac{1}{d-1}((d-1) ! / 2)^{\frac{2}{d-1}}\left(n^{\frac{2}{d-1}} \log n\right), \quad n \rightarrow \infty
$$

and

$$
n(\lambda) \sim 2((d-1) !)^{-1}\left(\frac{|\log \lambda|}{\log |\log \lambda|}\right)^{\frac{d-1}{2}} .
$$

2.5. Sign-definite radial symbols with compact support. We introduce the following notion.

Definition 2.4. Let the function $V(r), r \geq 0$, have a compact support. The number $b$ is called the exact support radius (ESR) for $V$ if $V(r)=0$ for $r>b$, while for any $b^{\prime} \in(0, b)$,

$$
\int_{b^{\prime}}^{b}|V(r)| d r>0
$$

Proposition 2.5. Suppose that $b>0$ is the ESR for $V \geq 0$. Then for the operator $T_{V}$ in the spaces $\mathcal{B}_{\mathbf{R}}^{\mathbb{C}}, \mathscr{B}_{\mathbf{R}}^{\mathbb{R}}, \mathcal{B}_{\mathbf{R}}^{\mathbf{H}}, \mathscr{B}^{\mathbb{C}}, \mathscr{B}^{\mathbb{R}}, \mathscr{B}^{\mathbf{H}}, \mathscr{B}^{\mathbf{A H}}$ hold the asymptotic formulas (2.5), respectively, (2.7), (2.7), (2.16), (2.17), (2.17), and (2.20) (as well as the corresponding asymptotic formulas for the counting function.)

Proof. In all cases, the asymptotic estimate from above is trivial, since the numerator in the expression for the eigenvalues $\Lambda_{k}$ increases when $V$ is replaced by the characteristic function of the ball with radius $b$, multiplied by some positive constant, and this constant is not felt in the logarithmic scale. As for the lower estimates, the reasoning is similar for all cases. We present it, as an example, for operator in the space $\mathscr{B}_{\mathbf{R}}^{\mathbb{C}}$.

For the operator $T_{V}$ in $\mathscr{B}_{\mathbf{R}}^{\mathbb{C}}$, fix some $b^{\prime}<b$. We have

$$
\begin{aligned}
\int_{0}^{b} V(r) r^{2 k+2 d-1} d r & \geq \int_{b^{\prime}}^{b} V(r) r^{2 k+2 d-1} d r \\
& \geq\left(b^{\prime}\right)^{2 k+2 d-1} \int_{b^{\prime}}^{b} V(r) d r .
\end{aligned}
$$

Passing to the logarithmic scale, we obtain

$$
\log \left(\lambda_{n}^{+}\right)=\log \left(s_{n}\left(T_{V}\right)\right) \gtrsim n^{1 / d}(d !)^{\frac{1}{d}} \log \left(b^{\prime} / \mathbf{R}\right),
$$

which gives the required lower asymptotic estimate, due to the arbitrariness of $b^{\prime}$. 


\subsection{Rapidly decaying sign-definite symbols}

Definition 2.6. A bounded function $V(r), r \in[0, \infty)$, is called rapidly decaying, $V \in \mathcal{R} \mathscr{D}$, if

$$
V(r)=o\left(\exp \left(-r^{\varsigma}\right)\right), r \rightarrow \infty \text { for any } \varsigma>0,
$$

or, equivalently, $\log |V(r)|<-C r^{\varsigma}, C>0$, for any $\varsigma>0$.

For further reference, we formulate here an important property of functions in $\mathcal{R D}$, which is easily established by a proper change of variables.

Lemma 2.7. If $V \in \mathcal{R} D$, then

$$
\left|\int_{0}^{\infty} V(r) r^{s} d r\right|=O(\Gamma(\varepsilon s)), \quad s \rightarrow \infty,
$$

for any $\varepsilon>0$.

Proposition 2.8. Let $V \geq 0, V \in \mathcal{R} D$. Then for the eigenvalues of the operator $T_{V}$ in the spaces $\mathscr{B}^{\mathbb{C}}, \mathscr{B}^{\mathbb{R}}, \mathscr{B}^{\mathbf{H}}, \mathscr{B}^{\mathrm{AH}}$ the eigenvalue asymptotic formulas (2.16), respectively, (2.17), (2.17), and (2.20) hold (as well as the corresponding asymptotic formulas for the counting function.)

Proof. Consider the complex Bargmann space first. If $V \in \mathcal{R} \mathscr{D}, V \geq 0$, the numbers $\Lambda_{k}^{\mathbb{C}}$ are given by the same formula (2.13). By monotonicity and Lemma 2.7,

$$
|\log | \int_{0}^{\infty} V(r) r^{k} d r||=o(k \log k), \quad k \rightarrow \infty
$$

and, therefore, for the numbers $\log \Lambda_{k}^{\mathbb{C}}$ we have the same asymptotics (2.14) as for a compactly supported symbol, which leads to the asymptotics (2.15) for the counting function of the operator $T_{V}$. The same reasoning takes care of the space $\mathscr{B}^{\mathbb{R}}$.

Consider now the operator in the space $\mathcal{B}^{\mathrm{H}}$. For a general $V \in \mathcal{R} \mathscr{D}$, for estimating the numerator in (2.19), we use the representation formula for Bessel functions,

$$
\begin{aligned}
J_{v}(r)=\left(\frac{r}{2}\right)^{v} & {[\Gamma(v+1 / 2) \Gamma(1 / 2)]^{-1} } \\
& \int_{-1}^{1}\left(1-t^{2}\right)^{v-1 / 2} \cos (r t) d t, \quad \operatorname{Re} v>-\frac{1}{2},
\end{aligned}
$$

see, e.g., [7], 8.411.8. It follows from (2.24) that

$$
\left|J_{k+\frac{d-2}{2}}(r)\right| \leq C r^{k+\frac{d-2}{2}} \Gamma\left(k+\frac{d-1}{2}\right)^{-1} ;
$$

substituting this bound into (2.19), we obtain

$$
\Lambda_{k} \leq C 2^{k}\left(\Gamma\left(k+\frac{d+1}{2}\right)\right)^{-1}\left[\int_{0}^{\infty} V(r) r^{k+d} d r\right]^{2} .
$$


By Lemma 2.7, the integral in (2.25) is majorized by $\Gamma(\varepsilon(k+d))$ for any $\varepsilon>0$. So, in logarithmic scale,

$$
\log \Lambda_{k} \sim-\log \left(\Gamma\left(k+\frac{d+1}{2}\right)\right)+o(k \log k),
$$

which gives the same asymptotics for $\log \Lambda_{k}$, as in the case of $V$ with compact support. So, for $V \in \mathcal{R} D$ the same asymptotics (2.16)-(2.15) hold.

The same reasoning takes care of operators in the Agmon-Hörmander spaces.

Remark 2.9. The asymptotic formulas for $n(\lambda)$ are the same for a compactly sup-

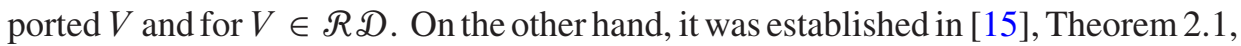
that if a reasonably regular $V$ does not belong to $\mathcal{R} D$, the asymptotics of $n\left(\lambda ; \mathscr{B}^{\mathbb{C}}\right)$ is different. This circumstance justifies the introduction of the class $\mathcal{R} D$. Further on, in Section 5 we consider oscillating symbols not belonging to $\mathcal{R} D$.

\section{Auxiliary theorems}

3.1. Reordering -2. As it was explained in the introduction, the main complication for proving lower estimates for eigenvalues lies in the need of reordering of the sequence of the eigenvalues $\Lambda_{k}$ obtained by the explicit formulas in Section 2. So, supposing that the lower estimate is wrong, and thus a contradicting upper estimate holds, we can obtain a bound for the reordered sequence of the numbers $\Lambda_{k}$, which, however, does not imply directly any estimate for the numbers $\Lambda_{k}$ themselves. In order to deal with this circumstance, we need the following statement which plays a key role in the sequel.

Proposition 3.1. Let $k \mapsto m_{k}$ be a bijection of the set of nonnegative integers $\mathbb{Z}_{+}$. For $\beta>1$, we denote by $E_{\beta}$ the set $\left\{k \in \mathbb{Z}_{+}: m_{k} \leq \beta k\right\}, F_{\beta}=\left\{m_{k}: k \in E_{\beta} \cdot\right\}$. Then

$$
\#\left\{F_{\beta} \cap[0, N]\right\} \geq \frac{\beta-1}{\beta} N,
$$

for any natural $N$.

In other words, Proposition 3.1 states that, under a bijection, a controllably nonzero share of integers $m_{k}$ are not too large, compared with $k$.

Proof. Suppose that $m_{k} \in[0, N] \backslash F_{\beta}$. Then $k<\frac{m_{k}}{\beta} \leq \frac{N}{\beta}$. And therefore $\#\left\{F_{\beta} \cap\right.$ $[0, N]\}>N+1-\frac{N}{\beta} \geq \frac{\beta-1}{\beta}(N+1)-\frac{1}{\beta}$.

Remark 3.2. The constant $\frac{\beta-1}{\beta}$ in Proposition 3.1 is sharp. In fact, set $m_{k}=[\beta k]+1$ for $k \in \mathbb{N} \backslash 2^{\mathbb{N}}$, while for integers powers of 2 we define $m_{k}$ so that to obtain a bijection. Then $\lim \sup N^{-1} \#\left\{F_{\beta} \cap[0, N]\right\}=\frac{\beta-1}{\beta}$. 
Proposition 3.1 leads to the following partial conversion of Proposition 2.1, mentioned in Section 2.1.

Proposition 3.3. Suppose that $a_{k}, b_{k}$ are real sequences, $b_{k}>0$ is non-increasing, and for the non-increasing permutation $a_{k}^{*}$ of $\left|a_{k}\right|$, we have

$$
a_{k}^{*} \leq b_{k}
$$

Then for any $\beta>1$ there exists a subsequence $a_{k_{l}}, k_{1}<k_{2}<\cdots$, such that

$$
\left|a_{k_{l}}\right| \leq b_{\left[k_{l} / \beta\right]}
$$

and $k_{l} \leq\left[\frac{\beta}{\beta-1} l\right]+1$.

The statement means that if the sequence $\left|a_{k}\right|$, after being non-increasingly reordered, satisfies some sort of monotonous estimate, then in the initial sequence there exists a controllably dense subsequence, for which a similar but slightly weaker estimate holds.

Proof. Let the non-increasing permutation of the sequence $\left|a_{k}\right|$ be given by the bijection $j \mapsto m_{j}:\left|a_{m_{j}}\right|=a_{j}^{*}$, so that $\left|a_{m_{j}}\right| \leq b_{j}$. Thus, for any $m_{j} \in F_{\beta}$, we have $m_{j} \leq j \beta$, therefore $j \geq m_{j} / \beta$ and $b_{j} \leq b_{\left[m_{j} / \beta\right]}$. Now we take as the subsequence $k_{l}$, the elements $m_{j} \in F_{\beta}$ taken in the increasing order. The inequality (3.1) is therefore fulfilled. By Proposition 3.1, \#\{F $\cap[0, N]\} \geq \frac{\beta}{\beta-1} N$ for any $N$, which is equivalent to the second inequality we need.

We will also need a simple consequence of Proposition 3.1 concerning the rate of divergence of the series composed of the inverse values of $m \in F_{\beta}$.

Proposition 3.4. Under the conditions of Proposition 3.1,

$$
\limsup _{N \rightarrow \infty}(\log N)^{-1} \sum_{m \in F_{\beta} \cap[0, N]} m^{-1} \geq \frac{\beta-1}{\beta} .
$$

3.2. Estimates for functions analytical in a half-plane. There are a number of results in the classical complex analysis relating the estimates along the real axis of a function analytical in the half-plane $\operatorname{Re} \zeta>0, \zeta=\xi+i \eta$, with estimates of its values at some sequence of points. The first of such results we need, with ideas originating in [10], was obtained in [3], p. 200.

Theorem 3.5. Let $f(\zeta)$ be a function, analytical in the right half-plane, of exponential type, satisfying

$$
\int_{-\infty}^{\infty} \frac{\log _{+}|f(i \eta)|}{1+\eta^{2}} d \eta<\infty
$$


Suppose that $\mu_{l}$ is a monotone sequence of real points tending to infinity so that $\left|\mu_{l}-\mu_{l-1}\right| \geq \delta>0$ and $\sum \mu_{l}^{-1}=\infty$. Then

$$
\limsup _{l \rightarrow \infty} \frac{\log \left|f\left(\mu_{l}\right)\right|}{\mu_{l}}=\limsup _{\xi \rightarrow+\infty} \frac{\log |f(\xi)|}{\xi} .
$$

Remark 3.6. In [10] and in [3] the additional condition $\lim l \mu_{l}^{-1}=0$ was imposed. However, it was shown in [9] that this condition is excessive and can be deleted.

Theorem 3.5 will be used for the study of the spectrum of operators in Bergman spaces. For the case of Bargmann spaces another result about estimates of functions, not of exponential type, will be used. We cite its version from [4], see also [5], with an obvious typo corrected.

Theorem 3.7. Let the function $f(\zeta)$ be analytical in the half-plane $\operatorname{Re} \zeta=\xi>0$ and satisfy the estimate

$$
\begin{aligned}
& \left|f\left(\rho e^{i \varphi}\right)\right|=O(\exp [\rho(a \log \rho \cos \varphi+ \\
& \pi c|\sin \varphi|+b \cos \varphi)]), \quad|\varphi|<\pi / 2, \quad \rho \rightarrow \infty,
\end{aligned}
$$

for some $a \geq 0, c \geq-a / 2$. Suppose also that the growing sequence $\mu_{l}$ of positive numbers satisfies $\mu_{l+1}-\mu_{l} \geq \delta>0$ and

$$
\limsup _{N \rightarrow \infty}\left[\sum_{\mu_{l} \leq N} \mu_{l}^{-1}-(c+a / 2) \log N\right]=\infty .
$$

Then the bound

$$
\limsup _{l \rightarrow \infty} \frac{\log \left|f\left(\mu_{l}\right)\right|}{\mu_{l} \log \mu_{l}}<-2 c
$$

implies that $f(\zeta) \equiv 0$.

This theorem improves the classical result by N. Levinson, see [10], Theorem $\mathrm{XLI}$, in the sense that it does not require any regularity of the sequence $\mu_{l}$.

\section{Eigenvalues of Toeplitz operators with non-sign-definite symbols}

This section contains the main results of the paper. These results can be expressed in the following way:

$$
\limsup _{\lambda \rightarrow 0} \frac{n\left(\lambda ; T_{V}\right)}{n\left(\lambda ; T_{|V|}\right)}=1,
$$

in all spaces under consideration, where the radial function $V$ has compact support for $\Omega=D_{\mathbf{R}}$, and $V \in \mathcal{R} D$ for the case of $\Omega=\mathbb{C}^{d}$ or $\Omega=\mathbb{R}^{d}$. Further on, we consider the concrete cases in detail. 
4.1. Operators in Bergman spaces. In this section the symbol $V$ is supposed to be an arbitrary real bounded radial function with $\operatorname{ESR} b<\mathbf{R}$.

Theorem 4.1. For the singular numbers of the operator $T_{V}$ in the Bergman spaces the following asymptotic formulas hold.

For the complex space $\mathcal{B}_{\mathbf{R}}^{\mathbb{C}}$,

$$
\limsup _{\lambda \rightarrow 0} n(\lambda)|\log \lambda|^{-d}=(d !)^{-1}(2|\log (b / \mathbf{R})|)^{-d}, \quad n(\lambda)=n\left(\lambda ; T_{V}, \mathscr{B}_{\mathbf{R}}^{\mathbb{C}}\right) .
$$

For the harmonic and Helmholtz spaces $\mathscr{B}_{\mathbf{R}}^{\mathbb{R}}$ and $\mathscr{B}_{\mathbf{R}}^{\mathbf{H}}$ :

$$
\limsup _{\lambda \rightarrow 0} n(\lambda)|\log \lambda|^{-d}=2((d-1) !)^{-1}(2|\log (b / \mathbf{R})|)^{-d+1} ;
$$

for $n(\lambda)=n\left(\lambda ; T_{V}, \mathscr{B}_{\mathbf{R}}^{\mathbb{C}}\right) \operatorname{or} n(\lambda)=n\left(\lambda ; T_{V}, \mathscr{B}_{\mathbf{R}}^{\mathbf{H}}\right)$.

Proof. The proofs for the analytical and harmonic cases are almost identical; we present the first one.

The upper estimate in (4.2) follows from Proposition 2.5 by monotonicity and Proposition 2.1. We will prove the lower estimate. Suppose that it is wrong; this means that

$$
n\left(\lambda ; T_{V}, \mathcal{B}_{\mathbf{R}}^{\mathbb{C}}\right)<\gamma(d !)^{-1}(2|\log (b / \mathbf{R})|)^{-d}|\log \lambda|^{d}
$$

for some $\gamma<1$ and for $\lambda$ small enough.

The singular numbers of $T_{V}$ are equal to the numbers $\left|\Lambda_{k}\right|$ defined in (2.3), permuted in the non-increasing order (we denote by $\sigma_{m}$ this permuted sequence), with multiplicities $\mathbf{d}_{k}^{\mathbb{C}}$ given in Section 2. So, $\sigma_{m_{k}}=\left|\Lambda_{k}\right|$, where $k \mapsto m_{k}$ is some bijection of $\mathbb{Z}_{+}$.

By (2.4),

$$
n(\lambda)=\sum_{\left|\Lambda_{k}\right|>\lambda} \mathbf{d}_{k}^{\mathbb{C}}=\sum_{\sigma_{m_{k}}>\lambda} \mathbf{d}_{k}^{\mathbb{C}} .
$$

Since the numbers $\mathbf{d}_{k}^{\mathbb{C}}$ increase with $k$ growing, the quantity in (4.5) can only decrease if we replace in (4.5) the values of $k$ by their smallest possible values, i.e.,

$$
n(\lambda) \geq \sum_{k=0}^{n_{0}(\lambda)} \mathbf{d}_{k}^{\mathbb{C}}
$$

where $n_{0}(\lambda)=\#\left\{j: \sigma_{j}>\lambda\right\}$.

So, since $\mathbf{d}_{k}^{\mathbb{C}}=\left(1+O\left(k^{-1}\right)\right) k^{d-1}((d-1) !)^{-1}$ for large $k$, we have

$$
n(\lambda) \geq \sum_{k=0}^{n_{0}(\lambda)} \frac{k^{d-1}\left(1+O\left(k^{-1}\right)\right)}{(d-1) !} \geq\left(1+O\left(n_{0}(\lambda)^{-1}\right)\right) \frac{n_{0}(\lambda)^{d}}{d !} .
$$


Substituting (4.6) into (4.4), we obtain

$$
n_{0}(\lambda) \leq\left(1+O\left(|\log \lambda|^{-1}\right)\right) \gamma^{\frac{1}{d}}|\log \lambda|, \quad \text { or } \quad \sigma_{m} \lesssim\left(\gamma^{\prime} \frac{b}{\mathbf{R}}\right)^{2 m},
$$

for $\lambda$ small enough, resp., $m$ large enough, and some $\gamma^{\prime}<1$. Our next aim is to derive an estimate for $\Lambda_{k}$ from (4.7).

We fix some $\beta>1$, to be determined later, and apply Proposition 3.3 to the sequences $a_{k}=\left|\Lambda_{k}\right|$,

$$
b_{k}=\left(\gamma^{\prime} \frac{\beta}{\mathbf{R}}\right)^{2 m} .
$$

Thus there exists a subsequence $\Lambda_{k_{l}}$ such that

$$
\left|\Lambda_{k_{l}}\right| \leq C\left(\gamma^{\prime} \frac{b}{\mathbf{R}}\right)^{2 k_{l} \beta^{-1}} .
$$

We are going to show now that inequality (4.8) holds not only for the subsequence $\Lambda_{k_{l}}$ but for all $\Lambda_{k}$, probably with slightly worse constants. To do this, we introduce the complex variable $\zeta=\xi+i \eta$ and consider the function

$$
f(\zeta)=(2 \zeta+2 d) R^{-(2 \zeta+2 d)} \int_{0}^{\mathbf{R}} V(r) r^{2 \zeta+2 d-1} d r .
$$

The function $f(\zeta)$ is analytical and bounded in the half-plane $\operatorname{Re} \zeta=\xi>0$, so the condition (3.2) is satisfied. The values of $f$ at integer points $k$ coincide with the numbers $\Lambda_{k}$, due to (2.3). By the second inequality in (4.7), the series $\sum\left(k_{l}\right)^{-1}$ diverges. So, all conditions or Theorem 3.5 are fulfilled and, therefore,

$$
\limsup _{k \rightarrow \infty} \frac{\log \left|\Lambda_{k}\right|}{k}=\limsup _{l \rightarrow \infty} \frac{\log \left|\Lambda_{k_{l}}\right|}{k_{l}}
$$

or, returning back from the logarithmic scale,

$$
\Lambda_{k} \leq C\left(\gamma^{\prime} \frac{b}{\mathbf{R}}\right)^{2 k / \beta^{\prime}}
$$

for any $\beta^{\prime}>\beta$. Since $\gamma^{\prime}<1$, we can choose the parameter $\beta$ and then $\beta^{\prime}>\beta>1$ in the above reasoning so close to 1 that

$$
\left(\gamma^{\prime} \frac{b}{\mathbf{R}}\right)^{2 / \beta^{\prime}}<\left(\theta \frac{b}{\mathbf{R}}\right)^{2}
$$

for some $\theta<1$. We substitute (2.3) into (4.9) and obtain

$$
\int_{0}^{\mathbf{R}} V(r) r^{2 k+2 d-1} d r=O\left((\theta b)^{2 k}\right) .
$$


It remains to apply a classical theorem about the properties of the moments problem, say, Theorem 6.9.5 in [3], saying that (4.10) implies supp $V \subset[0, \theta b]$, and this inclusion contradicts our assumption that $b$ is the ESR for $V$.

Now we pass to the proof for the Helmholtz case. Again, suppose that (4.3) is wrong; this means that

$$
n\left(\lambda ; T_{V}, \mathcal{B}_{\mathbf{R}}^{\mathbf{H}}\right)|\log \lambda|^{-d+1}<2 \gamma((d-1) !)^{-1}(2|\log (b / \mathbf{R})|)^{-d+1}
$$

with some $\gamma<1$, for small $\lambda$. In the same way as for the case of the complex spaces, (4.11) implies the estimate for the numbers $\sigma_{m}$, the monotonically reordered sequence of the numbers $\Lambda_{k}=\Lambda_{k}^{\mathrm{H}}$ :

$$
\sigma_{m} \leq\left(\gamma \frac{b}{\mathbf{R}}\right)^{2 m}
$$

We can further proceed as before, to derive, by means of Proposition 3.3, from (4.12) the following estimate for a sufficiently dense subsequence in $\Lambda_{m}$ :

$$
\left|\Lambda_{k_{l}}\right| \leq C\left(\gamma \frac{b}{\mathbf{R}}\right)^{2 k_{l} / \beta^{\prime}}
$$

with $k_{l} \leq \frac{\beta}{\beta-1} l$.

Next, as before, we need to carry over the estimate (4.13) from the subsequence to the whole sequence $\Lambda_{k}$, and even to the fractional $k$. To achieve this, we consider the auxiliary function $f(\zeta)$ analytical in the half-plane $\xi=\operatorname{Re} \zeta>0$ :

$$
f(\zeta)=\Gamma(\zeta+d / 2) \Gamma(\zeta+(d+2) / 2)) \int_{0}^{\mathbf{R}} V(r) J_{\zeta+\frac{d-2}{2}}^{2}(r) r d r .
$$

By the known asymptotics of Bessel functions for a large index, see (2.11), the function $f(\zeta)$ is bounded in the half-plane $\operatorname{Re} \zeta>0$, moreover, by (2.9), (2.10), and (2.12), its values the integer points $\zeta=k$ are asymptotically equal to the numbers $\Lambda_{k}$.

Therefore, we can apply Theorem 3.5 to the function $f(\zeta)$, similarly to the reasoning for the complex case above, thus obtaining for real $\xi>0$ and some $\gamma^{\prime} \in(\gamma, 1)$

$$
|f(\xi)| \leq C\left(\gamma^{\prime} \frac{b}{\mathbf{R}}\right)^{2 \xi / \beta}, \quad \xi \rightarrow \infty,
$$

which means

$$
\begin{aligned}
& \int_{0}^{\mathbf{R}} V(r) J_{\xi+\frac{d-2}{2}}^{2}(r) r d r \\
& \quad \leq C[\Gamma(\xi+d / 2) \Gamma(\xi+(d+2) / 2)]^{-1}\left(\gamma^{\prime} \frac{b}{\mathbf{R}}\right)^{2 \xi / \beta}, \quad \xi \rightarrow \infty .
\end{aligned}
$$

Now we need to consider the cases of even and odd $d$ separately. 
For the case of an even dimension $d$, thus having an integer $\frac{d-2}{2}$, we write (4.14) for integer values $\xi=k$ :

$$
\begin{aligned}
& \int_{0}^{\mathbf{R}} V(r) J_{k+\frac{d-2}{2}}(r)^{2} r d r \\
& \quad \leq C[\Gamma(\kappa+d / 2) \Gamma(k+(d+2) / 2)]^{-1}\left(\gamma^{\prime} \frac{b}{\mathbf{R}}\right)^{2 k / \beta} .
\end{aligned}
$$

We use now C. Neumann's formula, see [20], 2.72(2), or [7], 8.536.2,

$$
\sum_{j=m}^{\infty} \frac{\Gamma(m+j)}{j \Gamma(j-m+1)} J_{j}^{2}(r)=\frac{(2 m) !}{(m !)^{2}}\left(\frac{r}{2}\right)^{2 m}, m \in \mathbb{Z}_{+} .
$$

It is easy to see from the Stirling formula that the series in (4.16) converges uniformly on finite intervals, and, asymptotically in $m$, the leading term prevails. We substitute the expression for $r^{2 m}$ with $m=k+\frac{d-2}{2}$ from (4.16) into $\int_{0}^{\mathbf{R}} r^{2\left(k+\frac{d-2}{2}\right)} V(r) d r$ :

$$
\int_{0}^{\mathbf{R}} r^{2 m} V(r) r d r=2^{2 m} \frac{(m !)^{2}}{(2 m) !} \sum_{j=m}^{\infty} \frac{j \Gamma(m+j)}{\Gamma(j-m+1)} \int_{0}^{\mathbf{R}} V(r) J_{j}^{2}(r) r d r .
$$

For each term in (4.17), we apply the estimate (4.15). Calculations with $\Gamma$-functions show that

$$
\int_{0}^{\mathbf{R}} r^{2 k} V(r) r^{d-1} d r=O\left(\left(\gamma^{\prime} b\right)^{2 k / \beta}\right)
$$

Finally, since $\gamma^{\prime}<1$, we can choose $\beta>1$ so close to 1 that $\left(\gamma^{\prime} b\right)^{1 / \beta}<\theta b, \theta<1$. Now we can again apply Theorem 6.9.5 in [3], which gives supp $V \subset[0, \theta b], \theta<1$ which contradicts our assumption that $b$ is the ESR for $V$.

A similar reasoning works for case of an odd dimension $d$. We however consider (4.14) for half-integer $\xi, \xi=k+1 / 2$. Then, since $(d-2) / 2$ is half-integer, (4.14) gives an estimate of integrals containing Bessel functions with integer index. The final step in the proof is the same.

4.2. Operators in Bargmann and $\mathbf{A H}$-spaces. For the case of Bargmann and $\mathrm{AH}$ spaces, the consideration follows the same idea as for the Bergman spaces, with main difference that the auxiliary analytical function $f(\zeta)$ will not be of exponential type any more.

Theorem 4.2. Let the radial symbol $V$ belong to $\mathcal{R} D$. Then

(i) for $\mathscr{B}=\mathscr{B}^{\mathbb{C}}\left(\mathbb{C}^{d}\right)$,

$$
\limsup _{\lambda \rightarrow 0}\left[n(\lambda)\left(\frac{|\log \lambda|}{\log |\log \lambda|}\right)^{-d}\right]=(d !)^{-1}
$$


(ii) for $\mathscr{B}=\mathscr{B}^{\mathbb{R}}\left(\mathbb{R}^{d}\right)$, or $\mathscr{B}^{\mathbf{H}}\left(\mathbb{R}^{d}\right)$

$$
\limsup _{\lambda \rightarrow 0}\left[n(\lambda)\left(\frac{|\log \lambda|}{\log |\log \lambda|}\right)^{-d+1}\right]=2((d-1) !)^{-1} ;
$$

(iii) for $\mathscr{B}=\mathscr{B}^{\mathrm{AH}}\left(\mathbb{R}^{d}\right)$

$$
\limsup _{\lambda \rightarrow 0}\left[n(\lambda)\left(\frac{|\log \lambda|}{\log |\log \lambda|}\right)^{\frac{-d+1}{2}}\right]=2((d-1) !)^{-1} .
$$

Proof. All cases are handled in a similar manner. We give the proof of the part (i) of Theorem 4.2 in detail and then explain the changes needed for other cases.

The proof starts in the same way, as for Theorem 4.1. The estimate from above in (4.18) is already established. Suppose that the lower bound in (4.18) is wrong. This means that for some $\gamma<1$, the inequality

$$
n(\lambda)<\gamma(d !)^{-1}\left(\frac{|\log \lambda|}{\log |\log \lambda|}\right)^{d}
$$

holds for all sufficiently small $\lambda>0$. The singular numbers of the operator $T_{V}$ are equal to the numbers $\left|\Lambda_{k}\right|$, see (2.13), permuted in the non-increasing order, with multiplicities $\mathbf{d}_{k}^{\mathbb{C}}$ (defined in Section 2.3.1). So, these singular numbers equal $\sigma_{m_{k}}=\left|\Lambda_{k}\right|$, where $k \mapsto m_{k}$ is some bijection of $\mathbb{Z}_{+}$. By (2.1),

$$
n(\lambda)=\sum_{\left|\Lambda_{k}\right|>\lambda} \mathbf{d}_{k}^{\mathbb{C}}=\sum_{\sigma_{m_{k}}>\lambda} \mathbf{d}_{k}^{\mathbb{C}} .
$$

Since the multiplicities $\mathbf{d}_{k}^{\mathbb{C}}$ are non-decreasing as $k$ grows, the quantity (4.22) can only decrease if we replace in the sum in (4.22) the values of $k$ by their smallest possible values,

$$
n(\lambda) \geq \sum_{k=0}^{n_{0}(\lambda)} \mathbf{d}_{k}^{\mathbb{C}},
$$

where $n_{0}(\lambda)=\#\left\{\sigma_{m}>\lambda\right\}$. So, since $\mathbf{d}_{k}^{\mathbb{C}}=k^{d-1}((d-1) !)^{-1}\left(1+O\left(k^{-1}\right)\right)$, we have

$$
n(\lambda) \geq \sum_{k=0}^{n_{0}(\lambda)} \frac{k^{d}}{(d-1) !}\left(1+O\left(k^{-1}\right)\right)=\left(1+O\left(n_{0}(\lambda)^{-1}\right)\right) \frac{n_{0}(\lambda)^{d}}{d !} .
$$

We substitute (4.24) into (4.21) and obtain

$$
\gamma^{\prime} n_{0}(\lambda) \leq(1+o(1)) \frac{|\log \lambda|}{\log |\log \lambda|}, \quad \gamma^{\prime} \in\left(1, \gamma^{-\frac{1}{d}}\right) .
$$


We rewrite (4.25) in terms of an estimate for $\sigma_{m}$ :

$$
\sigma_{m} \lesssim \Gamma\left(\gamma^{\prime} m\right)^{-1} .
$$

We derive now an estimate for $\log \left|\Lambda_{k}\right|$ from (4.26). Fix some $\beta>1$, to be determined later, and apply Proposition 3.3 to the sequences $a_{k}=\left|\Lambda_{k}\right|, b_{k}=\Gamma\left(\gamma^{\prime} m_{k}\right)^{-1}$. Thus there exists a subsequence $\Lambda_{k_{l}}$ such that

$$
\left|\Lambda_{k_{l}}\right| \lesssim \Gamma\left(\gamma^{\prime} k_{l} / \beta\right)^{-1},
$$

or, in the logarithmic scale,

$$
\log \left|\Lambda_{k_{l}}\right| \leq-\gamma^{\prime} \beta^{-1} k_{l} \log \left(k_{l} / \beta\right)(1+o(1)) .
$$

Now we introduce the complex variable $\zeta=\xi+i \eta$ and consider in the half-plane $\xi>0$ the function

$$
f(\zeta)=2 \int_{0}^{\infty} V(r) r^{2 \zeta+2 d-1} e^{-r^{2}} d r .
$$

This function coincides with $\Lambda_{k} \Gamma(k+d)$ at the integer points $\zeta=k$. Therefore, by (4.27) at the points $\zeta=k_{l}$, the function $f(\zeta)$ satisfies

$\log \left|f\left(k_{l}\right)\right|<\log \Gamma\left(k_{l} / \beta\right)-\gamma^{\prime} \beta^{-1} k_{l} \log \left(k_{l} / \beta\right)(1+o(1)) \lesssim\left(1-\gamma^{\prime} / \beta\right) \log \Gamma\left(k_{l} / \beta\right)$.

By Lemma 2.7, for $\zeta=\rho e^{i \varphi},|\varphi|<\pi / 2$

$$
|f(\zeta)|=O\left(\int_{0}^{\infty}|V(r)| r^{2 \rho \cos \varphi+2 d-1} e^{-r^{2}} d r\right)=O(\Gamma(\varepsilon \rho \cos \varphi)), \quad \rho \rightarrow+\infty
$$

for any $\varepsilon>0$. Taking into account the asymptotics for the $\Gamma$-function for large real values of argument, we obtain that the estimate (3.3) is satisfied for any $a>0$ and $c>0$. Now, we choose $\beta>1$ so that $\frac{\gamma^{\prime}}{\beta}>1$. After that, we fix $a, c>0$ so small that $\frac{\beta-1}{\beta}>\frac{a}{2}+c$ and $2 c<\frac{\gamma^{\prime}}{\beta-1}$. Then, by Proposition 3.4, the sequence of integers $\left\{\mu_{l}\right\}$ obtained by the increasing reordering of the sequence $\left\{k_{l}\right\}$, satisfies the conditions (3.4) and (3.5). So, all conditions of Theorem 3.7 are satisfied, and we can conclude that $f(\zeta)=0$ for all $\zeta$.

It only remains to notice that $f(-\zeta)$ is the Mellin transform of the function $V(r) r^{2 d-1} e^{-r^{2}}$, and by the inversion theorem for the Mellin transform we conclude that $V(r) \equiv 0$, and this takes care of the proof for the complex Bargmann space.

The proof for the space $\mathscr{B}^{\mathbb{R}}$ follows the reasoning above, only with $d$ replaced by $d-1$.

Now we consider the operator in the Helmholtz Bargmann space, where some more changes are needed. 
Similar to (4.21), we suppose that (4.19) is wrong, and this would mean that

$$
n(\lambda) \leq \gamma 2((d-1) !)^{-1}\left(\frac{|\log \lambda|}{\log \mid \log \lambda}\right)^{d-1}
$$

for small $\lambda$ and some $\gamma<1$. By repeating the calculations in (4.22)-(4.24), we obtain for the distribution function $n_{0}(\lambda)$ (now of the numbers $\Lambda_{k}=\Lambda_{k}^{\mathbf{H}}$ ) an estimate of the form (4.25), with some $\gamma^{\prime}>1$. After that, we apply again Proposition 3.3 to obtain for a (sufficiently dense) subsequence $\Lambda_{k_{l}}$ the estimate (4.27). Starting from this point, the reasoning is somewhat different.

We introduce the complex variable $\zeta=\xi+i \eta, \xi \geq 0$, and consider the function

$$
f(\zeta)=\Gamma^{2}(\zeta+(d-2) / 2) \int_{0}^{\infty} J_{\zeta+\frac{d-2}{2}}^{2} V(r) e^{-r^{2}} r d r .
$$

This function is analytical in the half-plane $\xi>-\frac{1}{2}$. It follows also from (2.24) that

$$
|f(\zeta)| \leq C \int_{0}^{\infty} r^{2(\zeta+d / 2)}|V(r)| e^{-r^{2}} d r
$$

and by $(2.23), f(\zeta)$ satisfies

$$
|f(\zeta)|=O(|\Gamma(\varepsilon \zeta)|)
$$

for any $\varepsilon>0$ as $|\zeta| \rightarrow \infty, \operatorname{Re} \zeta>-\frac{1}{2}$. It follows that the function $f(\zeta)$ satisfies condition (3.3) of Theorem 3.7, with arbitrarily small positive values of $a$ and $c$.

After this property has been established, the proof follows the one for the complex Bargmann space. For real integer $\zeta=k$ we have $|f(k)| \asymp\left|\Lambda_{k}\right| \Gamma(k+(d-2) / 2)$. Therefore, by (4.25), the values of $f(\zeta)$ at the points $\zeta=k_{l}$ satisfy (4.28). We take $\beta>1$ so close to 1 that $\frac{\gamma^{\prime}}{\beta}>1$ and then fix $a$ and $c$ so small that $\frac{\beta-1}{\beta}>a+\frac{c}{2}$ and $2 c<\frac{\gamma^{\prime}}{\beta}-1$. Then the sequence of integers $\mu_{l}$ obtained by the increasing reordering of the sequence $k_{l}$, satisfies all conditions of Theorem 3.7, and therefore $f(\zeta) \equiv 0$. This means, in particular, that all numbers $\Lambda_{k}$ are zeros, therefore $T_{V}=0$. To prove that this implies $V=0$, we use (4.16) to express $\int_{0}^{\infty} r^{2 m} V(r) e^{-r^{2}} d r$ as a linear combination of the numbers $\Lambda_{k}$, all of them being equal to zero. Therefore we obtain that the function $g(\zeta)=\int_{0}^{\infty} r^{2 \zeta+2 d} V(r) e^{-r^{2}} d r$ takes zero values at all integer points. Finally, we note that, again by Lemma 2.7, the function $g(\zeta)$ satisfies the conditions of Theorem 3.7 with arbitrarily small positive $a$ and $c$ and therefore $g \equiv 0$. The proof that $V \equiv 0$ is again concluded by the inversion theorem for the Mellin transform.

The case of the space $\mathcal{B}^{\mathrm{AH}}$ is proved in the same way, with minimal changes.

\section{Less rapidly decaying symbols. Counterexamples.}

The results of Section 4 might lead to the impression that, probably, one should expect the absence of cancelation of the positive and negative parts of the symbol in a more 
general situation as well. The author was of such opinion for a certain time. However, the examples given in this section show that such impression is wrong.

We present here a construction of symbols that decay at infinity rather fast but not sufficiently fast to get into the $\mathcal{R} D$ class. This symbols oscillate very rapidly at infinity. We show that the s-numbers of the Toeplitz operator with symbol $V$ decay considerably faster than the ones for the operator with symbol $|V|$, so an analogy with theorems in Section 4 does not hold. In order to simplify the calculations, we restrict ourselves here to the operators in the space $\mathscr{B}^{\mathbb{C}}$ in the one-dimensional case, $d=1$. The same constructions work in any dimension, and for the spaces $\mathscr{B}^{\mathbb{R}}$ as well. We suppose that for other spaces under consideration a similar construction produces proper examples.

We consider the symbol

$$
V(r)=V_{p, q}(r)=e^{-r^{2 p}+r^{2}} \sin \left(r^{2 q}\right)
$$

with $p>1, q>p$.

Theorem 5.1. For the operator $T_{V}$

$$
\limsup _{\lambda \rightarrow 0} n\left(\lambda, T_{V}\right) \frac{\log |\log \lambda|}{|\log \lambda|} \leq \frac{q}{q-1},
$$

while

$$
n\left(\lambda, T_{|V|}\right) \sim n\left(\lambda, T_{V_{+}}\right) \sim n\left(\lambda, T_{V_{-}}\right) \sim \frac{p}{p-1} \frac{|\log \lambda|}{\log |\log \lambda|}, \quad \lambda \rightarrow 0 .
$$

The difference in coefficients in front of $|\log \lambda| / \log |\log \lambda|$ in (5.2), (5.3) transforms into a large difference in the decay order of the eigenvalues:

$$
\log s_{n}\left(T_{V}\right) \lesssim \frac{q(p-1)}{p(q-1)} \log s_{n}\left(T_{|V|}\right),
$$

so, in fact, a rather strong cancelation takes place.

Proof. In order to prove (5.3), we need to estimate the numerator in (2.13), i.e., the integral

$$
\mathbf{I}(k)=\int_{0}^{\infty} e^{-r^{2 p}+r^{2}} \sin \left(r^{2 q}\right) e^{-r^{2}} r^{2 k+1} d r .
$$

After the change of variables $t=r^{2 q}$, (5.5) transforms to

$$
\begin{aligned}
\mathbf{I}(k) & =(2 q+1)^{-1} \int_{0}^{\infty} t^{\frac{k+2}{q}-1} e^{-t^{p / q}} \sin t d t \\
& =(2 q+1)^{-1} \operatorname{Im} \int_{0}^{\infty} t^{\frac{k+2}{q}-1} e^{-t^{p / q}} \exp (i t) d t .
\end{aligned}
$$


We consider here $t$ as a complex variable and the whole expression (5.6) as the integral along the positive real half-line.

Now we replace the integration line by means of rotating it to the line $\arg t=\pi / 2$. The integral in (5.6) does not change due to the factor $e^{-t^{p / q}}$ which decays fast in the whole first quarter. So, by setting $t=i \tau, \tau \in(0, \infty)$, we have

$$
|\mathbf{I}(k)| \leq(2 q+1)^{-1} \int_{0}^{\infty} \tau^{\frac{k+2}{q}-1} e^{-\tau} d \tau=\Gamma((k+2) / q) .
$$

Finally, taking into account the expression for the denominator in (2.13) and Stirling's formula we arrive at (5.2).

To prove (5.3), we estimate the integral, say,

$$
\mathbf{I}_{+}(k)=\int_{0}^{\infty} t^{\frac{k+2}{q}-1} e^{-t^{p / q}} \sin _{+}(t) d t
$$

from below. To do this we consider the intervals $I_{j}=(2 \pi / 3+4 \pi j, 4 \pi / 3+4 \pi j)$. On each of these intervals, for $j$ large enough, $\sin (t)>\frac{1}{2}$. This inequality easily implies a lower estimate for $\mathbf{I}_{+}(k)$ :

$$
\begin{aligned}
\mathbf{I}_{+}(k) & \geq \sum_{j} \frac{1}{2} \int_{I_{j}} t^{\frac{k+2}{q}-1} e^{-t^{p / q}} \sin _{+}(t) d t \\
& \geq C \int_{0}^{\infty} t^{\frac{k+2}{q}-1} e^{-t^{p / q}} d t \\
& =C \Gamma\left(\frac{k+2}{p}\right),
\end{aligned}
$$

which leads to (5.3).

We do not state here that the estimate in (5.2) is sharp; to prove this would require some rather hard calculations. However, we present here a slightly different but less general class of fast oscillating symbols, where a sharp estimate can be established on the level of proper references.

We consider the symbol

$$
V_{p}(r)=e^{-r^{2 p}+r^{2}} \sin \left(2 r^{4 p}+r^{2 p}\right),
$$

where $p>1$ is an arbitrary real number.

Theorem 5.2. For the operator $T_{V}$ we have

$$
n(\lambda) \sim \frac{2 p}{2 p-1} \frac{|\log \lambda|}{\log |\log \lambda|}, \quad \lambda \rightarrow 0,
$$

while for the operators $T_{|V|}, T_{V_{+}}, T_{V_{-}}$we have

$$
n(\lambda) \sim \frac{p}{p-1} \frac{|\log \lambda|}{\log |\log \lambda|}, \quad \lambda \rightarrow 0 .
$$


Proof. Consider the numerator in (2.13), i.e. the integral

$$
\mathbf{I}(k)=\int_{0}^{\infty} e^{-r^{2 p}+r^{2}} \sin \left(\frac{1}{2} r^{4 p}+r^{2 p}\right) e^{-r^{2}} r^{2 k+1} d r .
$$

After the change of variables $t=r^{2 p},(5.11)$ transforms to

$$
\mathbf{I}(k)=(2 p)^{-1} \int_{0}^{\infty} t^{\frac{k+2}{p}-1} e^{-t} \sin \left(\frac{1}{2} t^{2}+t\right) d t .
$$

The integral in (5.12) can be found in [7], 4.966 (6):

$$
\mathbf{I}(k)=e^{\frac{1}{2}} \Gamma\left(\frac{k+2}{p}\right) \sin \left(\pi \frac{k+2}{p}\right) D_{-\frac{k+2}{p}}(\sqrt{2}),
$$

where $D_{v}$ is the parabolic cylinder function. We use now the asymptotic formula for $D_{v}$, see [11], 8.1.6.

$$
\begin{aligned}
D_{v}(z) \sim 2^{-\frac{1}{2}}\left(\exp \left(\frac{v}{2} \log (-v)\right)-v / 2-z \sqrt{-v}\right) & \\
& \left(1+O\left(|v|^{-\frac{1}{2}}\right)\right), \quad|v| \rightarrow \infty, \quad \arg -v<\pi / 2 .
\end{aligned}
$$

Now, we combine (5.13) and (5.14) to get

$$
\log |\mathbf{I}(k)| \sim \log \Gamma\left(\frac{k}{p}\right)-\log \Gamma\left(\frac{k}{2 p}\right) \sim \frac{k}{2 p} \log k .
$$

Finally, taking into account the asymptotics of the logarithm of the denominator in (2.13), we arrive at (5.9).

The estimate (5.10) for the sign-definite symbol is established in the same way as in Theorem 5.1.

Remark 5.3. The properties of the symbols considered above are in a sense parallel to the ones studied in Chapter 6 of [18], where the question on boundedness and compactness of Toeplitz operators with radial symbols in the classical Bergman spaces on the unit disk was considered. As it was found in [18], if a symbol $V(r)$ is sign-definite and unbounded in the mean value sense near $r=1$, then the operator $T_{V}$ fails to be bounded. However, being multiplied by a sufficiently fast oscillating factor, such symbol generates a bounded and even compact operator. 


\section{References}

[1] S. Agmon and L. Hörmander, Asymptotic properties of solutions of differential equations with simple characteristics. J. Anal. Math. 30 (1976), 1-38. MR 0466902 Zbl 0335.35013

[2] A. Alexandrov and G. Rozenblum, Finite rank Toeplitz operators: some extensions of D. Luecking's theorem, J. Funct. Anal. 256 (2009), 2291-2303. MR 2498766 Zbl 1165.47018

[3] R. P. Boas, Entire functions. Academic Press, New York, 1954. MR 0068627 Zbl 0058.30201

[4] V. Eiderman, Decrease on a sequence of points of a function holomorphic on a half plane. Sibirsk. Mat. Zh. 24 (1983), 180-192. English transl.: Siberian Math. J. 24 (1983), 304-315. MR 0695300

[5] V. Eiderman and M. Essen, Uniqueness theorems for analytic and subharmonic functions. St. Petersburg Math. J. 14 (2003) 889-952. Transl. from: Algebra Anal. 14 (2002), 1-88. MR 1965913 Zbl 1030.30033

[6] N. Filonov and A. Pushnitski, Spectral asymptotics of Pauli operators and orthogonal polynomials in complex domains. Comm. Math. Phys. 264 (2006), 759-772. MR 2217290 Zbl 1106.81040

[7] I. S. Gradshtein and I. M. Ryzhik, Tables of integrals, series and products. Academic Press, New York, 2007. MR 2360010 Zbl 0448.65002

[8] D. Luecking, Finite rank Toeplitz operators on the Bergman space. Proc. Amer. Math. Soc. 136 (2008), 1717-1723. MR 2373601 Zbl 1152.47021

[9] A. F. Leontiev, On the growth of an entire function on a sequence of points. Mat.Sborn. 96 (1975), 601-613. English transl.: Math. USSR-Sb. 25 (1975), 567-578. MR 0374425

[10] G. Levinson, Gap and density theorems, Amer. Math. Soc., Providence (RI), 1940. Zbl 0145.08003 JFM 66.0332.01 MR 0003208

[11] W. Magnus, F. Oberhettinger, and R. Soni, Formulas and theorems for the special functions of mathematical physics. Springer Verlag, Berlin etc., 1966. 3rd enlarged ed. Zbl 0143.08502 MR 0232968

[12] M. Melgaard and G. Rozenblum, Eigenvalue asymptotics for weakly perturbed Dirac and Schrödinger operators with constant magnetic fields of full rank. Commun. Partial Differ. Equations 28 (2003), 697-736. MR 1978311 Zbl 1044.35038

[13] O. Parfenov, Asymptotics of singular numbers of imbedding operators for certain class of analytic functions. Mat. Sb. 115 (1981), 632-641. English transl.: Math. USSR Sb. 43 (1982), 563-570. MR 0629631 Zbl 0473.47041 (original Russian) Zbl 0492.47026 (English transl.)

[14] A. Pushnitski and G. Rozenblum, On the spectrum of Bargmann-Toeplitz operators with symbols of a variable sign. To appear in J. Anal. Math. Preprint 2009 arXiv:0912.4486.

[15] G. D. Raikov and S.Warzel, Quasi-classical versus non-classical spectral asymptotics for magnetic Schrödinger operators with decreasing electric potentials. Rev. Math. Phys. 14 (2002), 1051-1072. MR 1939760 Zbl 1033.81038

[16] G. Rozenblum, Finite rank Toeplitz operators in Bergman spaces. In Around the research of Vladimir Maz'ya. III: Analysis and Applications, Springer Verlag, New York etc., and Tamara Rozhkovskaya Publisher, Novosibirsk, 2010, 331-358. MR 2664714 
[17] M. Shubin, Pseudodifferential operators and spectral theory. Springer Verlag, Berlin etc, 2001. Second ed. MR 1852334 Zbl 0980.35180

[18] N. Vasilevski, Commutative algebras of Toeplitz operators on the Bergman space. Birkhäuser, Basel, 2008. MR 2441227 Zbl 1168.47057

[19] R. Strichartz, Harmonic analysis as spectral theory of Laplacians. J. Funct. Anal. 87 (1989), 51-148. MR 1025883 Zbl 0694.43008

[20] G. N. Watson, A treatise on the theory of Bessel functions, Cambridge University Press, London, 1995. Second ed. MR 1349110 Zbl 0174.36202

Received December 17, 2010; revised March 2, 2011

Grigori Rozenblum, 1. Department of Mathematics, Chalmers University of Technology, Sweden. 2. Department of Mathematics, University of Gothenburg, Chalmers Tvärgatan, 3, S-412 96 Gothenburg, Sweden

E-mail: grigori@math.chalmers.se 